\title{
Ein Blick auf den diesjährigen UMEM-Kongress in Athen
}

\section{E. Kloter}

Trotz der den literarischen Finessen oft abträglichen Sprachenvielfalt - die Schwierigkeiten versuchen wir durch Prints im Original mit beigefügten Übersetzungen zu dämpfen - wagen sich immer mehr schriftstellerisch tätige Ärzte und Kolleginnen an diesen internationalen Kongress, den die Griechen im September 2001 in Athen mustergültig organisierten.

Korrespondenz:

Dr. med. Eduard Kloter

Kreuzbühlweg 18

CH-6045 Meggen
Auch fünf aus unserer Schweizer Vereinigung ASEM waren zu Lesungen dabei.

Das Weltgeschehen lässt uns schreibende - eben auch zeitbeschreibende - Ärzte nicht unberührt. So einigten wir uns einstimmig am 29. September auf einen mahnenden Appell zu einem nur wohlüberlegten und konsequenzbedachten Kampf gegen den zunehmenden Terror aller Art unter Einbezug seiner Genese. Ein Vergleich aus unserer Berufsarbeit lag nahe.

Leider sind Vernunft und völkerpsychologische Überlegungen in der Weltmachtpolitik wenig gefragt.

Hier unser Aufruf und ein paar Gedanken zum und ums Konferenzgeschehen.

\author{
UMEM \\ Le Président \\ UNION MONDIALE DES ECRIVAINS MEDECINS \\ WELTUNION DER SCHRIFTSTELLERÄRZTE \\ UNIONE MONDIALE DEGLI SCRITTORI MEDICI \\ THE WORLD-UNION OF WRITING PHYSICIANS
}

\section{UMEM appeals to all the statesmen}

Writing physicians - doctors from all over the world - meeting in Athens at their $45^{\text {th }}$ annual assembly, discussing "Reality and Myths", are very deeply concerned and in fear about the development of hate and revenge after the terrible terrorist attack on vulnerable symbols of globalisation and wealth, nearly "mythical signs" between the rich and the poor in an always more exploited world, which brought pain and suffering and death to so many. Our deep sympathy and condolence goes to the victims and America.

Well knowing by profession and experience, that this cancer said terrorism of all kind cannot be eradicated by simple hits of power somewhere or somehow without sophisticated diagnosis and profound thinking about the roots of the so called Evil and Good, we appeal to all statesmen and politicians to abstain from all sudden military activities creating even deeper splitting of to-days mankind.

Hellenic Society of Writing Physicians Prof. John Koliopoulos
UMEM Union mondiale des écrivains-médecins Dr. Edi Kloter 


\title{
$45^{\mathrm{e}}$ congrès UMEM des écrivains- médecins à Athènes, 26 au 30 septembre 2001
}

\author{
F. Verrey Bass
}

Pourquoi voler en trois heures jusqu'à Athènes, quand on peut y arriver autrement, beaucoup plus lentement, en ayant la possibilité de voir les paysages, d'apprécier, de côtoyer les gens? Voilà la raison pour laquelle d'Ancône nous avons pris le ferry jusqu'à Patras, de là la ligne de bus pour Athènes gare des bus. Le dernier trajet, nous l'avons fait en taxi. Le tout dit en deux lignes, mais depuis Bienne cela représenta deux jours et demi de trajet. Voyage réservé en août, bien avant le début de tous les problèmes et absolument rien qui pouvait annoncer ceux-ci. Mais on ne pourra jamais assez dire combien ce lent transfert est bénéfique, garantie de dépaysement complet, préparation judicieuse à vivre quelques jours dans une forme de médecine bien différente de celle que l'on connaît.

Le congrès a eu lieu en dehors d'Athènes, dans un hôtel à un jet de pierre de la mer, dans des conditions excellentes à tous points de vue, le temps étant bien

Correspondance:

Dr Françoise Verrey Bass

Quai du Bas 35

CH-2502 Bienne sûr de la partie. Il a fait très chaud, ce qui nous a permis d'apprécier cocktail de bienvenue et repas d'adieu encadré de danses folkloriques bien installés autour de la piscine. Notre hôte, le Professeur J. Koliopoulos, à l'instar de ses prédécesseurs, a réussi à nous communiquer en trois jours et demi les facettes les plus importantes, les plus caractéristiques de son pays.

Nous avons eu naturellement une très belle visite de la ville en car avec arrêts fort bien commentés sur les sites si connus que les énumérer serait une injure. Nous avons consacré une journée entière à une excursion inoubliable jusqu'à Delphes, l'un des plus beaux joyaux de ce pays si riche en culture antique, situé, ce qui rehausse encore sa beauté, dans une nature absolument exceptionnelle. Nous y étions à la fin de la saison touristique, un atout de plus, car moins de monde.

Cet instant d'arrêt et de contemplation est chaque année une vraie bouffée d'oxygène, car reçus par les collègues du pays qui invite, nous avons une occasion tout à fait spéciale de déguster si l'on peut dire non seulement la gastronomie du pays, mais aussi sa richesse intellectuelle à un niveau très différent de celui du touriste que nous sommes en général ailleurs. 